\title{
Using the Behaviour Change Wheel (BCW) to Characterise Parenting Interventions to Prevent Intergenerational Child Abuse
}

\author{
Fatima Younas $^{1}$ D $\cdot$ Leslie Morrison Gutman ${ }^{1}$ (D)
}

Accepted: 31 October 2021 / Published online: 28 November 2021

(c) The Author(s) 2021

\begin{abstract}
Research shows that parents with a history of child abuse are at risk of perpetuating the cycle of abuse; however, exploration of intervention content is still a neglected area. This qualitative study identifies intervention components and corresponding mechanisms of change of parenting interventions to prevent intergenerational child abuse. Interviews with ten heads of interventions from the UK and USA were coded using deductive framework analysis. The Behaviour Change Technique (BCT) Taxonomy and Behaviour Change Wheel were used to code intervention components including BCTs and intervention functions. Mechanisms of change were coded using the Theoretical Domains Framework. Twelve BCTs and eight intervention functions were identified including education, enablement and training delivered through BCTs of instruction on how to perform a behaviour, restructuring the environment and social support. Corresponding mechanisms of change include behaviour regulation, knowledge and social influences, among others. This study offers insight into targeting and tailoring services to improve outcomes for parents with a history of child abuse. Findings suggest that there are possible mechanisms through which vulnerable parents can be helped to break the cycle of abuse including promoting social support, regulating parents' behaviour through trauma-informed approaches and enhancing knowledge, self-esteem and confidence in parenting.
\end{abstract}

Keywords Behaviour Change Wheel $\cdot$ Intergenerational child abuse $\cdot$ Parenting interventions $\cdot$ Mechanisms of change $\cdot$ Intervention components $\cdot$ Behaviour change techniques

Leslie Morrison Gutman

1.gutman@ucl.ac.uk

Fatima Younas

Fatima.younas.15@ucl.ac.uk

1 Department of Clinical, Educational and Health Psychology, University College London, London, England 
Intergenerational child abuse is when individuals experience abuse as children and then later become abusive parents themselves. Although most individuals who were maltreated as children do not grow up to abuse their own children, research has generally found that rates of child abuse in families with one or more parents with a history of abuse are higher compared to the general population (Widom et al., 2015). Meta-analytic evidence from 142 studies (149 samples; 227,918 dyads) that underwent a methodological quality review, for example, revealed a significant but modest association of intergenerational maltreatment (Madigan et al., 2019). Engel (2004) describes the cycle of child maltreatment, "when a child is emotionally, physically or sexually abused it not only damages the child, but it damages the offspring of that child" (p. 1). Research contends that parents' ability to acknowledge and reflect on their own histories of childhood abuse can help reduce the risk of abuse to their own children (Dixon et al., 2009). Furthermore, it is also suggested that children who lack appropriate role models in their parents tend to develop inappropriate methods of conflict resolution as adults, have difficulties in interpersonal relationships and have heightened stress responses and impulsivity, all of which may increase the risk of perpetuating the cycle of maltreatment (Ornduff et al., 2001).

There is a vast literature which demonstrates the deleterious and long-standing consequences of child abuse (see Dahake et al., 2018 for a review). Given this, there is a proliferation of interventions that aim to prevent or reduce child abuse in highrisk families. Several studies have identified the intervention components of parenting interventions to prevent child abuse (Temcheff et al., 2018; Van der Put et al., 2017), including those focused on parent training interventions (Gubbels et al., 2019).

What has not been elucidated, however, are the specific intervention components (what is being delivered) and their corresponding mechanisms of change (how an intervention produces change) of parenting interventions to prevent intergenerational child abuse. Yet, parents with their own history of child abuse may benefit from distinct intervention strategies and require specific types of support to enable them to access and benefit from these interventions (Levey et al., 2017). For parents with a history of abuse, studies have identified the role of stress (Biaggi and Pariante, 2015; Morelli et al., 2020) and complex trauma (Chamberlain et al., 2019) as important processes explaining the intergenerational continuity of abuse. Parents with childhood histories of maltreatment who do continue the cycle of abuse may have an impaired capacity to both identify and reflect on their past traumatic experiences which ultimately impairs their ability to respond appropriately to their child's needs (Berthelot et al., 2015; George and Solomon, 2008). Intervention components, including the provision of practical, social and emotional support, strengthening the parent-child relationship and counselling with parents to address the trauma, have been utilised to break the cycle of abuse (Pasalich et al., 2019).

A systematic identification and understanding of intervention components that work in practice to break the cycle of child abuse is the first step to building an evidence base that informs both policy and practice in the field. As intergenerational child abuse consists of a constellation of abusive, neglectful and/or harmful behaviours in a specified population, this study employs a behavioural perspective to better understand possible mechanisms of change and intervention components that 
may help to break the cycle. This study uses the Behaviour Change Wheel (BCW) approach, which is a set of theory-based tools developed in behavioural science for characterising and designing behaviour change interventions (Michie et al., 2014). Using the BCW, the aim of this study is to identify and describe intervention components and their corresponding mechanisms of change through the examination of qualitative interviews with ten heads (developers) of parenting interventions focused on breaking the cycle of child abuse and neglect.

\section{Parenting Interventions}

The identification of intervention components to prevent or reduce child abuse and neglect is essential for designing new parenting interventions and improving existing ones (Gubbels, et al., 2019). In their review of common components of parenting interventions for preventing child maltreatment of school-aged children among at-risk families, Temcheff and colleagues (Temcheff et al., 2018) found that most evidence-based programs included components such as improving parent-child communication, regulating the emotions of parents, improving parenting skills and providing parent education.

There are no reviews examining intervention components of parenting interventions focused on preventing intergenerational child abuse, specifically. However, previous research suggests that enhancing parents' social support may be a key component. Numerous studies indicate that a lack of a supportive social network for parents contributes to the perpetuation of the intergenerational child abuse cycle (Berlin et al., 2008; Crouch et al., 2001). Individuals' history of child abuse can directly impact their ability to form trusting relationships and seek support from others. Possible mechanisms of change include improving parents' social skills and their interpersonal relationships to reduce their social isolation (Dixon et al., 2009).

Another intervention component is strengthening the attachment between parents and children through targeted behaviour change and video feedback. Research has established a link between parents' representations of their own childhood attachments and the attachment formed with their own infants (Zeanah et al., 1993). Parents' cognitive representations of attachment can be altered by forming stable and nurturing relationships in adulthood. Parenting interventions use relationship-based strategies and focus on promoting a secure attachment between parent and child to break the cycle of abuse (Sameroff et al., 2004). Meta-analytic evidence indicates that interventions focused on improving parenting behaviours and strengthening parent-child relationships can reduce the risk of the cycle of child abuse, with some populations such as adolescent first-time mothers and those experiencing intimate partner violence being more responsive to intervention (Levey et al., 2017).

Trauma-based counselling and therapeutic services can also be important intervention components to prevent intergenerational child abuse. Parenting interventions which include a 'trauma-informed' approach focused on counselling and therapeutic services have been shown to be effective in regulating parents' emotions, targeting their low self-concept and interpersonal disturbances arising from a history of child abuse (Anderson et al., 2018). In some cases, parents may have post-traumatic stress 


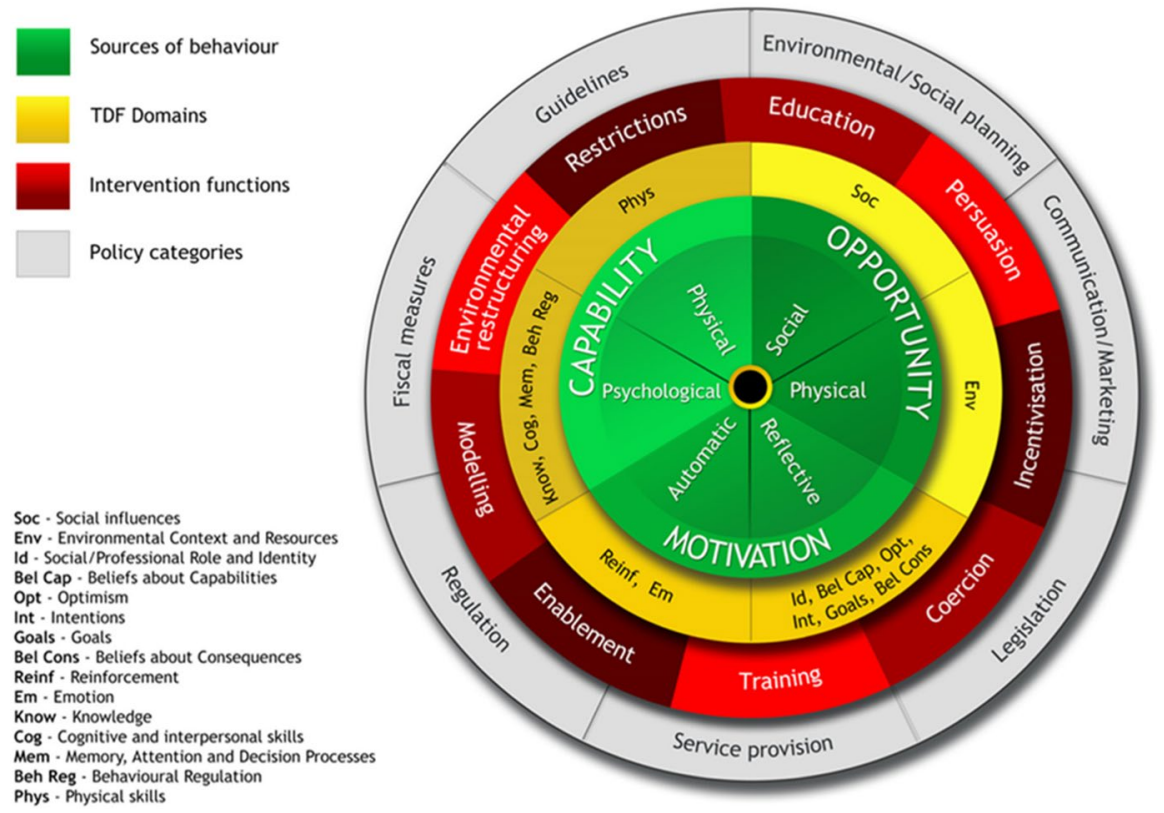

Fig. 1 Behaviour Change Wheel (BCW) and Theoretical Domains Framework (TDF) (Michie et al., 2011)

disorder, which can be addressed through trauma-based counselling (Muzik et al., 2013).

Overall, these studies suggest that there are a few intervention components, including enhancing social support, promoting a secure attachment between parent and child and counselling parents with trauma, which may be used in parenting interventions to prevent intergenerational child abuse. However, there is less systematic understanding regarding what specifically is being delivered in these interventions and the corresponding mechanism of change through which they can break the cycle of abuse. This can be addressed with the BCW framework, which provides an evidence-based methodology to understand and promote behaviour change (Michie et al., 2014). Using this approach, interventions can be better understood and potentially improved and replicated (Michie et al., 2014).

\section{Behaviour Change Wheel Framework}

The BCW is a systematic and validated approach derived from 19 other frameworks of behaviour change (Michie et al., 2011). The BCW can be used to identify what needs to change for the target behaviour to occur and provides information on the intervention components that can change behaviour (see Fig. 1).

The BCW consists of the COM-B model which includes capability (physical and psychological), opportunity (social and physical) and motivation (automatic 
and reflective). The Theoretical Domains Framework (TDF) breaks down COM-B into more detailed factors. These domains include factors at an individual level (e.g. knowledge and skills), social factors (e.g. social support) and environmental factors (e.g. environmental context and resources). This framework can be used to identify specific mechanism of change which can break the cycle of child abuse. The identification of mechanisms of change can help when further investigating what works, for whom and under what conditions (Kangaslampi \& Peltonen, 2019).

These domains can be linked to a further layer of the $\mathrm{BCW}$ which consists of nine intervention functions that can be used to enable behaviour change (Michie et al., 2011). Intervention functions are broad intervention components which describe how an intervention aims to change behaviour. For instance, education may be used by interventions to teach parents about child development and training equips them with positive parenting skills.

The Behaviour Change Technique (BCT) Taxonomy (BCTT, v1) collates a list of specific, evidence-based BCTs. BCTs are the 'active ingredients' of an intervention, specifying the more granular components. BCTs describe the way in which an intervention function is delivered, such as goals and planning, feedback and monitoring and social support. Expert consensus allows for mapping BCTs to intervention functions linked to the TDF domains (Michie et al., 2014). A BCT can be linked to several intervention functions; for example, the BCT of explaining health consequences of a certain behaviour can be linked to the intervention functions of education and persuasion. Similarly, a BCT can be linked multiple mechanisms of change; for example, the BCT of providing social support can be linked to social influences and behavioural regulation. Using this mapping, intervention components and their corresponding mechanisms of change are specified, allowing greater transparency in intervention improvement and replication.

\section{Present Study}

While previous reviews have investigated the components of parenting interventions to prevent child abuse, none of these have focused on the prevention of intergenerational abuse. A further limitation is that more specific intervention components which specify delivery (BCTs) and their corresponding mechanisms of change to end the cycle of child abuse are not clearly presented (MacMillan \& Wathen, 2014). As maltreating parents are a varied group with a multitude of differing needs, tailoring intervention strategies to better fit those with a history of childhood abuse may be necessary to prevent intergenerational child abuse (Levey et al., 2017). Childhood history of abuse among parents can also lead to enhancement of other risk factors including substance abuse, PTSD and other mental illnesses and can further push families into disadvantage and increase risk of child maltreatment (Levey et al., 2017). For interventions to incorporate specific strategies to break the cycle of abuse, an understanding of the mechanisms through which possible change may occur is essential. This study delineates some of these mechanisms with the potential to effectively break the cycle of abuse, which ultimately may inform interventions to better support parents with a history of abuse (Toth et al., 2015). 
Using the $\mathrm{BCW}$ and its associated tools, this qualitative study examines the intervention components and their corresponding mechanisms of change in ten parenting interventions to break the cycle of abuse. We focused on interventions within the USA and UK due to similarities in the countries in respect to the distribution of parenting interventions for child abuse and neglect. We included interventions both with and without evidence of effectiveness to better examine differences in the content and mechanisms of these programs. Based on interviews with 10 intervention heads, this study first identifies the intervention components (BCTs and intervention functions) and then examines their corresponding mechanisms of change using the TDF (Michie and Johnston, 2013). We used a qualitative method of investigation and mapping to identify linkages among BCTs, intervention functions and their hypothesised mechanisms of change.

\section{Method}

\section{Sample and Recruitment}

An online Google search (search words included parental interventions, intergenerational child abuse, breaking the cycle of abuse, parenting programs and child abuse prevention) was conducted and information about the intervention gathered from the websites and through email and phone contact. Authors are aware of the limitations of using Google to search for interventions including a lack of comprehensive and relevant results, unintentional bias in selecting interventions and issues surrounding quality of results obtained. While not a systematic method of searching, issues surrounding relevance and quality were mitigated by devising a search strategy which included specific key words for optimal search results and ensuring all information is retrieved from the intervention websites including a focus on the intergenerational component of child abuse. Furthermore, a Google search was considered necessary as interventions do not clearly state a focus on intergenerational child abuse and the majority of such interventions are labelled as child abuse interventions. For this reason, it was essential to go to each website and establish contact with the intervention through emails or phone calls to understand the extent of their focus on the prevention of intergenerational child abuse.

We included interventions within the UK or USA which had an intergenerational component. Initially, we located 23 interventions and shortlisted those fitting the criteria for selection. The inclusion criteria consisted of specific services within the intervention that targeted prevention of intergenerational child abuse and included participants (parents) with a history of childhood abuse. Five interventions did not fit the criteria as they did not include specific strategies to address intergenerational child abuse. We contacted the heads of the remaining 18 interventions via an initial email briefly explaining the purpose of the study and inquiring about their willingness to participate. From these, ten agreed to participate and written consent was obtained via email and an interview date and time scheduled. The interviews were conducted over the phone or Skype and audio-recorded. 
Table 1 (see Supplementary File) provides the characteristics of the parenting interventions in this study. Six of the ten interventions are based in the UK while the remaining four are in the USA. Length of the programs ranges from 8 months to 5 years and they are delivered to parents. Two of the interventions also include delivery to other family members. Five of the interventions are evidence-based with a published randomised control trial (RCT), and the remainder are categorised as without any evidence. Evidenced interventions have two types of evidence: (i) parenting outcomes related to risk and protective factors for child abuse and (ii) child abuse occurrence. Identifying details of the interventions and the names of interviewees are kept anonymous for five of the interventions as participants did not consent to share these details. For the remaining five, the interventions included NSPCC Together for Childhood (UK; intervention 1), Anna Freud Centre's Early Years Parenting Unit (UK; intervention 2), Stefanou Foundation (UK, intervention 3), Baby FAST (USA, intervention 8) and Triple-P (USA, intervention 9).

\section{Procedure}

Semi-structured interviews were guided by the COM-B model and questions were designed to fall under each of the three domains (capability, opportunity and motivation). An interview discussion guide (see Supplementary File) was used to explore intervention content including aims, mode of delivery and key mechanisms (social, psychological and environmental) which break the cycle of intergenerational child abuse and how the intervention delivers these (BCTs). Questions were asked about how the intervention facilitates participants' capability and motivation to break the cycle of abuse. For instance, to assess parents' psychological capabilities, the interview asked, "What psychological understanding do parents need to have to break the cycle of abuse? How does the intervention help with this understanding?" Further, questions about parents' motivation to break the cycle of abuse and how the intervention helps foster this motivation were asked. Lastly, interview questions were asked about the social and environmental factors that help break the cycle of abuse and how the intervention enables parents through strengthening these factors.

Respondents addressed BCTs through questions regarding ways in which the program is delivered and the specific outcomes that are targeted. For example, the interview asked respondents for specific examples on how parents' understanding is developed or how the program delivers support to parents.

Two pilot interviews were conducted with practitioners working in child abuse interventions in the UK, and some minor amendments in the interview discussion guide were made following the pilot interviews. The pilot interviews were not included in the study. The ten interviews included in the study lasted for around 45 min each and were audio-recorded and then transcribed.

\section{Data Analysis}

Interview analysis utilised a thematic (Braun \& Clarke, 2006) and deductive framework approach (coding against behaviour change frameworks). As a first step, the 
Fig. 2 Behaviour change techniques (BCTs), intervention functions and mechanisms of change for intergenerational child abuse interventions, a summary of findings

first author extracted the delivery strategies and then coded these against the BCTT, which were then linked to corresponding intervention functions using guidance provided in the Behaviour Change Wheel (Michie et al., 2014). The second step involved the first author extracting the corresponding mechanisms of change which were linked to each BCT in the interviews and then coding these using the TDF. Both steps were done manually on a spreadsheet.

The first author then tested the linkages between the BCTs and their corresponding mechanisms of change using The Theory and Techniques Tool (Carey et al., 2019), which was devised to synthesise behavioural interventions by identifying mechanisms of change through which BCTs have their effects. This online interactive tool depicts the strength of link between the mechanism of change and BCT through triangulation data from two studies: a literature synthesis and an expert consensus study. BCTs and mechanisms of change with evidence of 'no-link' were not reported (i.e. link was absent in literature synthesis study and experts in consensus study agreed that there was no link).

For the purposes of reliability, the second author coded two of the interviews. The authors established $82 \%$ reliability. Discrepancies were discussed, and changes were agreed. The second author further checked the other eight interviews for consistency.

\section{Ethics}

The study was approved (Ref: 13,479/002) by the University College London Research Ethics Committee.

\section{Results}

This section presents the findings in respect to BCTs, intervention functions and mechanisms of change. Table 2 (see Supplementary File) characterises the intervention components including the BCTs and BCW intervention functions and their corresponding mechanisms of change. Twelve BCTs and eight intervention functions were identified in the 10 interventions. TDF domains were linked to the intervention components and there were 12 mechanisms of change. The most commonly occurring BCTs, intervention functions and mechanisms of change across all interventions are presented in detail below (See Fig. 2 for a summary of the key findings).

\section{BCTs and Intervention Functions}

The most frequent BCT, instruction on how to perform a behaviour, occurred in eight of the 10 interventions. This was mostly linked to the intervention function of education and consisted of teaching parents about child development, appropriate 


\section{BCTs}

\begin{tabular}{|l|}
\hline Instruction on How to \\
Perform a Behaviour - \\
Mentalisation-based \\
therapy, emotional self- \\
regulation, strategies for \\
parents to deal with \\
negative emotions, \\
group/individual counselling, \\
trauma-informed practices, \\
teaching parents about child \\
development, behavioural \\
strategies (e.g., discipline) \\
and classes on child-parent \\
attachment \\
Reducing Negative \\
Emotions - Helping parents \\
manage triggers of stress \\
and anger \\
\end{tabular}

\section{Intervention Functions}

\begin{tabular}{|c|}
\hline Education \\
Enablement \\
Training \\
\hline
\end{tabular}

Mechanisms of Change

\section{Knowledge - Child}

development, child discipline and behavioural strategies

Behavioural Regulation -

Healing the parents' inner

child and regulating

emotions

Skills - Learning skills for

coping, managing difficult

behaviour and regulating

stress and parenting skills

(e.g. disciplining child)

\section{BCT}

\begin{tabular}{|l|}
\hline Restructuring the Social \\
and Physical \\
Environment - Linking \\
education services, \\
employment, housing, \\
links with support centres \\
and local community \\
services, media campaign \\
to promote awareness \\
Social Support (Practical, \\
Emotional, Unspecified) - \\
Support groups with \\
other members of the \\
family, Relationship \\
building with the \\
practitioner, home \\
visiting services, pre-natal \\
involvement with \\
families, parents' \\
discussion groups, \\
trauma-informed work \\
with parents, \\
motivational interviewing
\end{tabular}

Intervention Functions

\begin{tabular}{|c|}
\hline $\begin{array}{c}\text { Environmental } \\
\text { Restructuring }\end{array}$ \\
Education \\
Enablement \\
Training
\end{tabular}

Mechanisms of Change

Social Influences -

Enabling parents to form

nurturing relationships

Environmental Context and Resources -

Accessing practical

services and links with

community

\section{BCTs}

\section{Intervention Functions}

Information about Antecedents and Information about

Emotional Consequences -

Teaching parents about own feelings, self-soothing, toolkits to understand children's behaviour, minimising challenges and increasing help-seeking

Goal Setting (Behaviour and Outcome) - Pre-screening and assessments for developin strategies, discussions at beginning of intervention to set goals and implement changes, assessment to discuss social services' concerns and assessing parents' capacity for change

Monitoring of Behaviour and Feedback on Behaviour - Video interaction guidance to help with child-parent interaction and home visitation

Reattribution - Helping parents understand the effects of coercive/punitive parenting

Training
Mechanisms of

Change

Goals - Setting goals of

behaviour change and

enhancing self-esteem

Behavioural Consequences

and Behavioural Capability

Coping with adverse

circumstances/history,

motivational strategies to

enance confidence in

parenting 
interactions with their child and strengthening their parenting skills. Interventions utilised this BCT through antenatal parenting modules. For example (intervention 3), "We explain to our parents how babies' brains develop and why this period from conception to age two is so important". Interventions also focused on strengthening the parent-child relationship by teaching strategies for managing their child's behaviour. For instance (intervention 4), "We teach parents how to change their child's behaviour like look them in the eye, not yelling at them, putting the phone down and giving them attention".

Restructuring the environment (social and physical) was the second most frequent $\mathrm{BCT}$, found in seven interventions. These were linked to the intervention functions of enablement and environmental restructuring. An example of this BCT is a media campaign to raise awareness about parenting difficulties. For example (intervention 4), "A media campaign where you normalise help-seeking behaviours, de-stigmatise parenting education". Interventions, utilising the function of environmental restructuring, also focused on creating a community where parents could easily seek help and support. For instance (intervention 5), "places like family support centres and these universally available things are very powerful". One intervention utilised the BCT of restructuring the physical environment through starting "information and education initiatives in schools, in community centers and campaigns... in collaboration with the police locally or the local authority or the health trust locally" (intervention 1) to enhance awareness in communities so they are equipped to spot early signs of child abuse and neglect and have easy access to services for families requiring support.

Social support (practical, emotional and unspecified) was also a commonly occurring BCT and found in six of the 10 interventions. This BCT was linked to the intervention functions of enablement and environmental restructuring. Within this BCT, interventions focused on providing social support to parents through home visitation and by linking them to community support such as substance abuse recovery programs (practical), building a trusting relationship between the parents and practitioner (emotional) and becoming involved with families prenatally or in the newborn period to provide preventative intervention (unspecified). For instance (intervention 9), "We will link them to support services if there is domestic abuse... or we can refer them to a child psychologist if there are any behavioural issues...we link them to employment services and help them to get back to educational courses". In another example (intervention 5), "Through home visitation type services... when parents are in the attachment phase. When babies are very young, their families may require support and to help them with positive parenting strategies". Furthermore, a few interventions encouraged parents to participate in community activities such as a book club or fund-raising for a charity in a bid to create a "network of those that need help and helpers" (intervention 7).

Monitoring of behaviour by others was found in five interventions and linked to the intervention functions of restriction and enablement. Monitoring often included home visiting and/or follow-up visits. For example (intervention 8), "We can and sometimes do follow up visits with parents who we find are most at risk just to see how they are getting on after they've finished the program with us". 
Goal setting (behaviour) was found in four interventions and linked to the intervention functions of persuasion and enablement. This BCT was used to enable parents to set parenting goals and feel a sense of accomplishment by achieving them. For example (intervention 8), "we, with the parent, help them to set goals and seek help to eradicate the issues they face". This often led to enhanced self-esteem and more confidence in parenting. For example (intervention 3), "[Achieving goals] is really heart-warming for the parents and gives the parent self-belief in their parenting".

Feedback on behaviour was also found in four interventions and mostly linked to the intervention functions of training and modelling. One intervention used video recordings of parent-child interactions and viewed these later with the parent to highlight positive parenting instances. For example [intervention 3], “...show clips of good, attuned interactions and then you have a conversation with the parents". Another intervention asked parents to keep a record of their parenting behaviours and issues they faced in a journal (intervention 8). "Then we ask them to bring this journal and we review their behaviour and equip them with better coping mechanisms". Often interventions used the initial assessment to provide feedback to parents about behaviours that need changing, for instance (intervention 2), “... assessment for capacity to change and that's the parents' ability to address the concerns from social services".

Information about emotional consequences was found in four interventions and linked to the intervention functions of enablement and training. This BCT entailed provision of therapeutic services in the form of trauma centred counselling; for instance (intervention 8), "so they can speak about their own childhoods and if there was abuse there, which is often the case, then the healing begins". Interventions enabled parents to reflect on the effects of childhood abuse on their parenting behaviour and parent-child relationship through therapy and counselling support.

The BCT of information about antecedents was found in three interventions and linked to the intervention functions of education, training and enablement. For this BCT, there was a focus on tapping into the motivation of parents to improve their parenting and then helping them to devise strategies for coping with and managing the difficulties of parenting. For example (intervention 5), "helping parents to build that tool kit such as .... understanding what the different reasons are why the baby may be crying", and for instance (intervention 5), "teaching them how and why they [parents] feel the way they do".

Reducing negative emotions was found in three interventions and linked to the intervention function of enablement. Helping parents to regulate their emotions of anger, stress and sadness, especially in relation to their own childhood abuse, was a key component of this BCT. For example (intervention 6), "Certainly, we know that parents who have come from that place where they feel more victim than in control...they are operating from a survival perspective and have more difficulty regulating emotions...". Furthermore, interventions enabled parents to identify the cause of their negative emotions, for example (intervention 3), "understand their triggers and manage those triggers".

The less frequent BCTs across the interventions included reattribution, monitoring of emotional consequences, information about health consequences, salience of 
consequences, focus on past successes and commitment which were all identified in only two interventions while the BCTs of goal setting (outcome), behavioural practice/rehearsal, verbal persuasion about capability, reframing and punishment only occurred in one intervention each.

One notable finding is some of the more punitive intervention functions such as restriction, punishment and coercion were minimal in occurrence compared to the other intervention functions. Restriction was used by one intervention in which parents were encouraged to limit and cut off ties with those family members that increased stress and negatively impacted parents' ability to care for their child. Coercion was also not a common intervention function but was used by one program in which parents' participation and attendance in the program was mandated by a court order with the threat of escalation of child protective services. Furthermore, punishment was used by one intervention as a looming threat of children being taken away if parents are unable to make and sustain changes in their behaviour.

\section{Mechanisms of Change}

Social influences was mentioned as a mechanism of change in the 10 interventions. It was frequently targeted through enablement using BCTs of social support and restructuring the social environment. A key component of this domain consisted of enabling parents to form nurturing relationships with intervention practitioners, other adults/parents in the community and their own family members. For example (intervention 8), "We do intensive work with these parents over a long period of time, sometimes going on for over a year depending on the severity of their problems. So, they begin to view us as friends and helpers and see it as a support system-something which is lacking for many parents - the availability of a 'fall back' person and of knowing that they can talk to and get help from someone else". In another example (intervention 6), "We also found that safe, stable and nurturing relationships between adults (including other adults in the community) could also protect kids in the next generation".

The second most frequent mechanism of change was behavioural regulation, which was identified in nine of the ten interventions and mostly linked to BCTs of social support and monitoring of behaviour by others. It was also commonly linked to the intervention functions of training and enablement and less frequently linked to education. Behavioural regulation entailed interventions providing trauma-informed counselling to parents and building a strong, trusting relationship with them. For example (intervention 8), counselling enabled parents to speak about their childhood abuse, "...so the healing begins for that child the parent is carrying within themselves. If the inner child is healed, then the parent is able to adequately provide the nurturing for their own offspring". In another instance (intervention 3), "We have parents holding onto hurt and anger in families so the relationship between practitioner and service-users [parents] enable the parents to tell their story and help them to have compassion for themselves...to have compassion for their own parents... is key to breaking the cycle". 
Knowledge was another frequently occurring domain in nine of the ten interventions and mostly linked to BCTs of information on how to perform a behaviour and restructuring the social environment as well as the intervention functions of education, enablement and training. Interventions focused on enhancing parents' knowledge about child development, appropriate parenting strategies and even knowledge about their own behaviours and feelings and their effect on their parenting. For example (intervention 4), "A lot of the parents come in with problems of child behaviour and we help the parents to realise that as they change their behaviour, they change their children's behaviour". In another instance (intervention 6), “...we speak on behalf of the babies so the parents can realise that even stresses in their own lives that don't seem in their minds to be related to the infant development or infant health is having an impact on the infant".

The domain of goals in five interventions was mostly linked to the intervention functions of enablement and training and BCTs of goal setting and social support. Parents were encouraged to set goals of behaviour change, and achieving these goals enhanced the self-esteem and self-worth of parents. For example (intervention 2), "And this is just the beginning for them to make those changes to then go on for, hopefully if all goes well and if they want it and we want, in an ideal world, for those changes to be sustained and consistent. Both for them as its their mental health that they are struggling with as well as for their children".

Beliefs about consequences was also found in five of the ten interventions and linked to the intervention functions of enablement and training and BCTs of social support and information about emotional consequences. Within this domain, interventions enabled parents to cope with their past childhood abuse and adverse circumstances. For example (intervention 4), "Life can be very difficult in light of the things that have happened in the past, that you haven't processed or are avoiding processing, or you are so overwhelmed that you are feeling angry or upset all the time, to then be able to slow down and think about that...they learn in the 18 months.... enjoy being with your child rather than, you know, being terrified by them".

Beliefs about capabilities was identified in five interventions and linked to the intervention functions of enablement, training and modelling and BCTs of feedback on behaviour and focus on past successes. Interventions relied on motivating strategies as well as ways to enhance parents' self-worth for them to acquire more confidence in themselves as parents. In one example (intervention 8), "When parents feel recognised and feel that they have value and are able to do good, they then become motivated to change some of their negative behaviours and this reflects on their ability to successfully undergo programs or interventions and encourages them to seek help".

The most infrequently occurring domains were memory, attention and decision processes, identified in four interventions, intention in three interventions, while reinforcement was found in only two interventions. 


\section{Evidence-based Interventions Versus Non-evidence-based Interventions}

There were no differences noted in respect to intervention content between interventions with evidence and those without. The majority of the BCTs, intervention functions and mechanisms of change (TDF domains) overlapped between these two types of interventions. However, a few mechanisms of change were found more frequently in one compared to the other. For example, goals and behaviour regulation were found to be more common in evidence-based interventions, occurring frequently in all five of the evidence-based interventions while occurring only in one non-evidence-based intervention. Memory, attention and decision processes were more frequent in non-evidenced interventions, occurring in three of the five non-evidence based interventions. For the BCTs, punishment only occurred once in an intervention without any available evidence of effectiveness and reframing also occurred only once in an evidence-based intervention. For the intervention functions, incentivisation occurred only in one intervention with no evidence of effectiveness, while the other intervention functions occurred in at least one or more interventions showing evidence of effectiveness.

\section{Intervention Location}

The only significant difference noted between interventions in the UK and USA was those in the USA were more likely to have a home visiting component; three of the four interventions included either home visiting or follow-up visits with parents which were reflected in BCTs of social support and monitoring of behaviour, mechanisms of change including behaviour regulation and social influences and the intervention function of enablement. All six interventions in the UK did not include a home visiting component.

\section{Discussion}

Findings identified intervention components and their corresponding mechanisms of change in ten parenting interventions to understand possible levers that may drive behaviour change and disrupt the intergenerational cycle of child abuse. Twelve BCTs and eight intervention functions were characterised with the main mechanisms of change including behaviour regulation, knowledge, social influences and beliefs about capabilities, among others. Findings are discussed in light of the most frequent mechanisms of change noted in the interventions and how they correspond with BCTs and intervention functions. Implications about findings and limitations of the study are also presented.

\section{Behaviour Regulation (Psychological Capability)}

Based on the participants' perspectives, interventions aimed to equip parents to regulate their behaviour mostly through developing insight into parents' past abuse 
and how this impacts their current parenting behaviours and interactions with their child. Motivational interviewing was one technique that some of the interventions incorporated in their strategies, allowing parents to express ambivalence about change through eliciting statements that bring out their motivation to change behaviours (Holland \& Holden, 2016) and providing them with information about the origins and management of stress (BCT of information about antecedents). Pears and Capaldi (2001) state that parents with a history of abuse experience a great deal of stress when dealing with their children's behaviour resulting in a greater likelihood of abusive behaviour. Maternal distress has also been shown to be a significant mediator of the association between mothers' history of abuse and their use of abusive parenting strategies (Biaggi and Parenti, 2015; Morelli et al., 2020). Research has further found that parents with a history of abuse, especially those who physically abuse their children, have poor control over their aggressive impulses (Seng \& Prinz, 2008). Therefore, equipping parents with ways of regulating their impulses, emotions and behaviours can help disrupt the cycle of child abuse. In this study, interventions aimed to monitor parent-child interactions (BCT of monitoring of behaviour by others) to help parents regulate their behaviour and practice attuned and appropriate parenting.

Another way in which interventions aimed to equip parents to regulate their behaviours was through the intervention function of environmental restructuring and BCT of social support (practical and unspecified). The availability of parenting support groups, participation in community activities and creation of a network of parents and helpers within the local community allowed parents to feel at ease, discuss any issues and garner support. Previous research targeting community-based prevention efforts supports the effectiveness of initiatives such as improving neighbourhood contexts and allowing parents to rear their children in supportive communities to reduce the risk of child abuse (Daro \& Dodge, 2009; Zimmerman \& Mercy, 2010).

\section{Knowledge (Psychological Capability)}

This study found that increasing parents' knowledge was one of the ways in which participants mentioned to target disrupting the cycle of child abuse. Interventions aimed to enhance this knowledge by helping parents understand child development and age-appropriate expectations of their children based on their stage of development. Participants revealed that interventions focused on teaching positive parenting skills. Research has found that the risk of child abuse was reduced among mothers who were abused in childhood through enhancing their knowledge about child development and improving their parenting skills (Olds et al., 1997). Interventions aimed to increase this knowledge by teaching parents (BCT of instruction on how to perform a behaviour) through lectures, seminars, discussion groups and workshops.

Interventions also taught parents about attributing emotions (through the BCT of reattribution) appropriately to children's behaviour. For instance, some parents may feel a child cries to make them upset rather than out of hunger. Abusive parents compared to non-abusive parents attribute more hostility to their children's behaviour, 
and past research shows that parents' negative perception of their children's behaviour is a strong predictor for physical abuse (Young et al., 2018). Hence, interventions equipping parents with knowledge of child development and age-appropriate behaviours could help parents break the cycle of abuse.

\section{Social Influence (Social Opportunity)}

This study also found that social influences played a key role in interventions with the aim of helping mitigate the effects of parental childhood abuse and minimising the risk of future abuse. Social influences refer to the environmental influences that can impact parents' behaviour. Participants revealed that this influence is likely to be achieved through building a trusting relationship between parents and practitioners delivering the intervention. This links to previous studies of intergenerational continuity of abuse which have shown that parents' ability to form and maintain trusting relationships with other adults and social support from family, friends and the wider community, including support from an intervention, can significantly reduce the risk of child abuse (Martin et al., 2012). Some interventions also focused on strengthening relationships and easing conflict between parents and their family members and friends. The interventions addressed this through parent and family groups and by involving family members in projects such as cooking together and arts and crafts. Prior studies have demonstrated the positive role of safe, stable and nurturing relationships in breaking the cycle of child abuse (Jaffee, et al., 2013; Dixon et al., 2009). The present study's findings correspond with previous research and further reinforce the hypothesised role of the social environment in lowering child abuse risk and the need for intervention strategies that enhance mechanisms of change which focus on parents' social environment.

The majority of interventions included in the study incorporated intervening strategies to socially influence parents' behaviour through providing social support (BCT) to parents such as helping with issues like housing and employment and linking them to community or medical support services such as substance abuse recovery. Research suggests a strong link between parental stress and child abuse and helping parents with contextual factors that may contribute to their stress can significantly reduce that risk (Jackson, 2009). Additionally, research finds that individuals who have a supportive social network can cope more effectively with stress (Yoon, 2013). Expanding parents' social network by facilitating parents to participate in community activities and connecting them to other parents may also help in reducing parents' social isolation, a significant risk factor in child abuse (Tucker \& Rodriguez, 2014). Prior research shows that the lack of a supportive network contributes to continuation of the cycle of child abuse (Berlin et al., 2008; Crouch et al., 2001). Abusive parents are often cut off from social resources (Gracia \& Musitu, 2003), and social influences can act as a buffer to moderate the effects of other child abuse risk factors such as parenting stress (Crouch et al., 2001). 


\section{Belief About Capabilities (Reflective Motivation)}

Positive enhancement of parents' beliefs about their own parenting capabilities was also focused upon by interventions. One intervention modelled (intervention function) positive parenting by showing parents video clips of their attuned interactions with their child and discussing these behaviours with the parents. This formed part of providing feedback to the parents (BCT of feedback on behaviour) to help them practice appropriate ways of parenting.

Some interventions emphasised parents' past successes (BCT) and used persuasion and training (intervention functions) to facilitate the development of parents' positive parenting skills. Parents who feel comfortable in their parenting role show increases in their confidence and self-esteem and a lowered risk of perpetration of the cycle of abuse (Macmillan et al., 2009). Prior research shows that interventions which target parents' low self-concept and interpersonal disturbances arising from a history of child abuse are effective in breaking the cycle of abuse (Anderson et al., 2018). Evidence also shows that a strength-based approach, such as providing positive feedback and focusing on parents' strengths, is linked with increased parenting competency and higher engagement with the intervention which, in turn, reduces the risk of continuing the cycle of child abuse (Greene et al., 2004; Huebner et al., 2006).

\section{Implications of Findings}

The BCW framework has allowed encapsulation of key components of intergenerational parenting interventions by systematically highlighting BCTs and intervention functions that may help programs to break the cycle of child abuse. A key component of interventions within this study that sets them apart from other child abuse interventions is the provision of tailored strategies that focus on parents' trauma from their child abuse histories (Lucassen, et al., 2011). One of the ways interventions in this study aimed to accomplish this was by linking parents' past child abuse to their current negative parenting behaviour and then targeting behaviour change to break the cycle. Interventions used trauma-informed ${ }^{1}$ practices including counselling and therapy to address past childhood abuse and its effect on parenting behaviours (Wilkes, 2002). These practices create stability and safety for parents through relationship-strengthening approaches (with practitioners and other family members; Bysom, 2001), enabling parents feel a sense of control, empowerment and parenting confidence, which includes helping them to set and achieve goals. Parenting interventions which have a 'trauma-informed' approach focused on counselling and therapeutic services have also been shown to be effective in regulating parents' emotions (Anderson et al., 2018).

Parents' motivation to be 'better parents' (McWey et al., 2013) and not to repeat the cycle of abuse also needs to be addressed by interventions. It is important

\footnotetext{
1 Strength-based framework which is based on understanding and responding to the impact of trauma with the aim of building a sense of control and empowerment for the victim (Hopper et al., 2010).
} 
to recognise that parents often have adverse circumstances (e.g. mental health issues, domestic violence, unemployment) compounded by trauma from childhood adversity but are nevertheless doing their best to fulfil their parenting role in very difficult circumstances. This knowledge can help interventions to be non-judgmental and supportive, specifically tailoring programs to better support parents with a history of being abused as a child.

\section{Limitations}

As this study is a first step in using the BCW to unpack parenting interventions to prevent child abuse, it is an exploratory study with limitations. One limitation is that the study did not conduct interviews with parents to understand their experiences. The study relied on professionals (namely, intervention heads or providers) to provide an account of what is delivered, how and why; this may not fully capture any issues with implementation, reach, delivery and its effect on parenting outcomes. Further, response bias from interviewees cannot be ruled out due to their association with the interventions. Another limitation of the study is the small sample of interventions (10 interviews) from the USA and UK only. It is possible that some mechanisms of change, BCTs and/or intervention functions may not have been captured fully in the interviews and are not applicable to interventions in other countries. A small sample size also threatens the generalisability of results. While the sample size in this study is relatively small, literature suggests that in qualitative research, the sample size should be large enough to allow encapsulation and understanding of new phenomenon but small enough so that thorough analysis of data collected can be undertaken (Morse, 2000; Ogden \& Cornwell, 2010). Considering the exploratory nature of the current study, the quality, richness and usability of the data collected from interviews remained the primary focus and the authors felt that data collected from the ten interviews were sufficient to elucidate the aims of the current study.

Due to its exploratory nature, one key limitation is that it is difficult to elucidate the specific components that are most effective in breaking the cycle of abuse because findings rest on the perceptions, opinions and views of the intervention developers included in the study. However, this study is a first step towards identifying effective components of intergenerational interventions that can help break the cycle of abuse and further studies with a larger sample that also incorporate the perspectives of service-users can help validate and expand this study's findings and shed further light on the content and mechanisms of change in parenting interventions to prevent intergenerational child abuse.

\section{Conclusions}

This study offers insight into targeting and tailoring services to improve outcomes for parents with a history of child abuse. The findings suggest that there are key mechanisms through which vulnerable parents can be helped to break the cycle 
of abuse. Specifically, data from this study highlight the importance of promoting social support, regulating parents' behaviour through trauma-informed approaches and enhancing their knowledge, self-esteem and confidence in parenting.

The $\mathrm{BCW}$ is a useful tool that can be applied to parenting interventions to understand and change parents' behaviours to disrupt the cycle of child abuse. For example, while previous research highlights the importance of enhancing parents' knowledge and education about child development, this study provides further clarity on how knowledge can be provided by interventions (through education and training), what purpose it serves (enhances psychological capability) and why it is useful (allows parents to have age-appropriate expectations of child) in reducing the risk of intergenerational child abuse. The BCW approach thus allows for a systematic breakdown of intervention content which provides insight not only into what interventions need to focus on but also how and why the focus should be on a particular function or strategy, facilitating the translation of research into more effective practice.

Supplementary Information The online version contains supplementary material available at https://doi. org/10.1007/s42448-021-00103-8.

\section{Declarations}

Ethics Approval The study was approved (Ref: 13479/002) by the University College London Research Ethics Committee.

Conflict of Interest The authors declare no competing interests.

Open Access This article is licensed under a Creative Commons Attribution 4.0 International License, which permits use, sharing, adaptation, distribution and reproduction in any medium or format, as long as you give appropriate credit to the original author(s) and the source, provide a link to the Creative Commons licence, and indicate if changes were made. The images or other third party material in this article are included in the article's Creative Commons licence, unless indicated otherwise in a credit line to the material. If material is not included in the article's Creative Commons licence and your intended use is not permitted by statutory regulation or exceeds the permitted use, you will need to obtain permission directly from the copyright holder. To view a copy of this licence, visit http://creativecommons.org/licen ses/by/4.0/.

\section{References}

Anderson, K., \& van ur, E. (2018). Mothers and children exposed to intimate partner violence: A review of treatment interventions. International Journal of Environmental Research and Public Health, 15(9), 1955.

Berthelot, N., Ensink, K., Bernazzani, O., Normandin, L., Luyten, P., \& Fonagy, P. (2015). Intergenerational transmission of attachment in abused and neglected mothers: The role of trauma-specific reflective functioning. Infant Mental Health Journal, 36(2), 200-212.

Berlin, L. J., Appleyard, K., \& Dodge, K. A. (2008). Intergenerational continuity in child maltreatment: Mediating mechanisms and implications for prevention. Child Development, 82(1), 162-176.

Biaggi, A., \& Pariante, C. (2015). Intergenerational transmission of stress: Risk factors and underlying mechanisms. Giornale Di Psicopatologia, 21(1), 40-47. 
Braun, V., \& Clarke, V. (2006). Using thematic analysis in psychology. Qualitative Research in Psychology, 3(2), 77-101.

Bysom, T. L. (2001). Exceptions to the intergenerational transmission of violence: The role of perceived social support. Dissertation Abstracts International Section A: Humanities and Social Sciences, 61, 4564.

Carey, R. N., Connell, L. E., Johnston, M., et al. (2019). Behaviour change techniques and their mechanisms of change: A synthesis of links described in published intervention literature. Annals of Behavioural Medication, 53(8), 693-707.

Chamberlain, C., Gee, G., Harfield, S., Campbell, S., et al. (2019). Parenting after a history of childhood maltreatment: A scoping review and map of evidence in the perinatal period. PLoS One, 14(3).

Crouch, et al. (2001). Childhood physical abuse, early social support, and risk for maltreatment current social support as a mediator of risk for child physical abuse. Child Abuse \& Neglect, 25, 93-107.

Dahake, P., Kale, J. Y., \& Dadpe, M. V. (2018). Impact of child abuse and neglect on children: A review article. MIDSR Journal of Dental Research, 1(1), 36-42.

Daro, D., \& Dodge, K. (2009). Creating community responsibility for child protection: Expanding partnerships, changing context. The Future of Children, 19(2), 67-94.

Dixon, L., Browne, K., \& Hamilton-Giachritsis, C. (2009). Patterns of risk and protective factors in the intergenerational cycle of maltreatment. Journal of Family Violence, 24(2), 111-122.

Engel, B. (2004). Breaking the cycle of abuse: How to move beyond your past to create an abuse-free future. John Wiley \& Sons Inc.

George, C., \& Solomon, J. (2008). The caregiving system: A behavioural systems approach to parenting. In J. Cassidy \& P. R. Shaver (Eds.), Handbook of attachment: Theory, research, and clinical applications (2nd ed., pp. 833-856). Guilford Press.

Greene, B., McAllister, C., \& Tarte, J. (2004). The Strengths-Based Practices Inventory: A tool for measuring strengths-based service delivery in early childhood and family support programs. Families in Society, 85, 326-334.

Gracia, E., \& Musitu, G. (2003). Social isolation from communities and child maltreatment: A cross cultural comparison. Child Abuse \& Neglect, 27, 153-168.

Gubbels, J., van der Put, E. C., \& Assink, M. (2019). The effectiveness of parent training programs for child maltreatment and their components: A meta-analysis. International Journal of Environmental Research and Public Health, 16(13), 2404.

Holland, G. W. O., \& Holden, G. W. (2016). Changing orientations to corporal punishment: A randomized, control trial of the efficacy of a motivational approach to psycho-education. Psychology of Violence, 6(2), 233-242.

Hopper, E. K., Bassuk, E. L., \& Olivet, J. (2010). Shelter from the storm: Trauma-informed care in homelessness services settings. The Open Health Services and Policy Journal, 3, 80-100.

Huebner, R. A., Jones, B. L., Miller, V. P., Custer, M., \& Critchfield, B. (2006). Comprehensive family services and customer satisfaction outcomes. Child Welfare, 85(4), 691-714.

Jackson, T. L. (2009). The study of parenting stress and social support among African American mothers. Capella University.

Jaffee, R. S., Bowes, L., Quellet-Morin, I., et al. (2013). Safe, stable, nurturing relationships break the intergenerational cycle of abuse: A prospective nationally representative cohort of children in the United Kingdom. Journal of Adolescent Health, 55, S4-S10.

Kangaslampi, S., \& Peltonen, K. (2019). Mechanisms of change in psychological interventions for posttraumatic stress symptoms: A systematic review with recommendations. Current Psychology. https://doi.org/10.1007/s12144-019-00478-5

Levey, J. E., Gelaye, B., Bain, P., et al. (2017). A systematic review of randomised controlled trials of interventions designed to decrease child abuse in high-risk families. Child Abuse \& Neglect, 65, 48-57.

Lucassen, N., Tharner, A., Van Ijzendoorn, M. H., Bakermans-Kranenburg, M. J., Volling, B. L., Verhulst, F. C., et al. (2011). The association between paternal sensitivity and infant-father attachment security: A meta-analysis of three decades of research. Journal of Family Psychology, 25(6), 986-992.

MacMillan, HL, and Wathen, CN. (2014). Research brief: Interventions to prevent child maltreatment. PreVAiL: Preventing Violence Across the Lifespan Research Network. London, ON.

MacMillan, H. L., Wathen, C. N., Fergussen, D. M., et al. (2009). Interventions to prevent child maltreatment and associated impairment. The Lancet, 17, 250-266. 
Madigan, S., Cyr, C., Eirich, R., Fearon, R. P., Ly, A., Rash, C., \& Alink, L. R. (2019). Testing the cycle of maltreatment hypothesis: Meta-analytic evidence of the intergenerational transmission of child maltreatment. Development and Psychopathology, 31(1), 23-51.

Martin, A., Gardner, M., \& Brooks-Gunn, J. (2012). The mediated and moderated effects of family support on child maltreatment. Journal of Family Issues, 33, 920-941.

McWey, L. M., Pazdera, A. L., Vennum, A., \& Wojciak, A. S. (2013). Intergenerational patterns of maltreatment in families at risk for foster care. Journal of Marital and Family Therapy, 39, $133-147$.

Michie, S., van Stralen, M. M., \& West, R. (2011). The behaviour change wheel: A new method for characterising and designing Behaviour change interventions. Implementation Science, 6(1), 42.

Michie, S., \& Johnston, M. (2013). Behaviour change techniques. In M. D. Gellman \& J. R. Turner (Eds.), Encyclopaedia of behavioural medicine (pp. 182-187). Springer.

Michie, S., Atkins, L., \& West, R. (2014). The behaviour change wheel. A guide to designing interventions. Great Britain.

Morelli, N. M., Duong, J., Evans, M. C., Hong, K., Garcia, J., Ogbonnaya, I. N., \& Villodas, M. T. (2020). Intergenerational transmission of abusive parenting: Role of prospective maternal distress and family violence. Child Maltreatment, 1077559520947816.

Morse, J. M. (2000). Determining sample size. Qualitative Health Research, 10(1), 3-5.

Muzik, M., Ads, M., Bonham, C., et al. (2013). Perspectives on trauma-informed care from mothers with a history of childhood maltreatment: A qualitative study. Child Abuse \& Neglect, 37(12), 1215-1224.

Ogden, J., \& Cornwell, D. (2010). The role of topic, interviewee, and question in predicting rich interview data in the field of health research. Sociology of Health \& Illness, 32(7), 1059-1071.

Olds, D. L., Eckenrode, J., Henderson, C. R., et al. (1997). Long term effects of home visitation on maternal life course and child abuse and neglect: Fifteen year follow up of a randomised trial. JAMA, 278, 637-652.

Ornduff, S. R., Kelsey, R. M., \& O'Leary, K. D. (2001). Childhood physical abuse, personality and adult relationship violence: A model of vulnerability to victimization. American Journal of Orthopsychiatry, 3, 322-331.

Pasalich, D. S., Fleming, C. B., Spieker, S. J., Lohr, M. J., \& Oxford, M. L. (2019). Does parents' own history of child abuse moderate the effectiveness of the promoting first relationships intervention in child welfare? Child Maltreatment, 24(1), 56-65.

Pears, K. C., \& Capaldi, D. M. (2001). Intergenerational transmission of abuse: A two generational prospective of an at-risk sample. Child Abuse \& Neglect, 25, 1439-1461.

Sameroff, A. J., McDonough, S. C., \& Rosenblum, K. L. (Eds.). (2004). Treating parent-infant relationship problems: Strategies for intervention. Guilford Press.

Seng, A. C., \& Prinz, R. J. (2008). Parents who abuse: What are they thinking? Clinical Child and Family Psychology Review, 11, 163-175.

Temcheff, E. C., Letarte, M.-J., Boutin, S., \& Marcil, K. (2018). Common components of evidence-based parenting programs for preventing maltreatment of school-age children. Child Abuse \& Neglect, 80, 226-237.

Toth, S. L., Sturge-Apple, M. L., Rogosch, F. A., \& Cicchetti, D. (2015). Mechanisms of change: Testing how preventative interventions impact psychological and physiological stress functioning in mothers in neglectful families. Developmental Psychopathology, 27, 1661-1674.

Tucker, M., \& Rodriguez, C. M. (2014). Family dysfunction and social isolation as moderators between stress and child physical abuse risk. Journal of Family Violence, 29(2), 175-186.

Van der Put, C., Assink, M., \& Boekhout, N. (2017). Predicting child maltreatment: A meta-analysis of the predictive validity of risk assessment instruments. Child Abuse \& Neglect, 73, 71-88.

Widom, C. S., Czaja, S. J., \& DuMont, K. A. (2015). Intergenerational transmission of child abuse and neglect: Real or detection bias? Science, 347(6229), 1480-1485. https://doi.org/10.1126/science. 1259917

Wilkes, G. (2002). Abused child to non-abusive parent: Resilience and conceptual change. Journal of Clinical Psychology, 58, 261-276.

Yoon, Anderson S. (2013). The role of social support in relation to parenting stress and risk of child maltreatment among Asian American immigrant parents. Doctorate in Social Work (DSW) Dissertations, 32. University of Pennsylvania.

Young, A., Pierce, M. C., Kaczor, M. S., Lorenz, D. J., et al. (2018). Are negative/unrealistic parent descriptors of infant attributes associated with physical abuse? Child Abuse \& Neglect, 80, 41-51. 
Zeanah, C. H., Benoit, D., Barton, M., Regan, C., Hirshberg, L., \& Lipsitt, L. (1993). Representations of attachment in mothers and their one-year-old infants. Journal of the American Academy of Child and Adolescent Psychiatry, 32, 278-286.

Zimmerman, F., Mercy, J. A. (2010). A better start. Child maltreatment prevention as a public health priority. Zero to three: The National Centre for Infants, Toddlers and Families. Washington, DC.

Publisher's Note Springer Nature remains neutral with regard to jurisdictional claims in published maps and institutional affiliations. 